114 MONITORING FOR HYPOGLYCAEMIC NEWBORNS SHOULD WE EXPAND OUR RISK CATEGORIES?

${ }^{1} \mathrm{M}$ Flavin, ${ }^{2} \mathrm{H}$ Osiovich, ${ }^{3} \mathrm{~K}$ Coughlin, ${ }^{4} \mathrm{~J}$ Ray, ${ }^{5} \mathrm{~L} \mathrm{Hu}$, ${ }^{6} \mathrm{~J}$ Andres Leon, ${ }^{4} \mathrm{M}$ Sgro, ${ }^{1} \mathrm{~A}$ Gallipoli, ${ }^{1} \mathrm{~K}$ Gregoire, 'L Barr, ${ }^{1} \mathrm{~K}$ Grewal. 'Paediatrics, Queen's University, Kingston, Canada; ${ }^{2} B C$ Children's Hospital, Vancouver, Canada; ${ }^{3}$ London Health Sciences Centre, London, Canada; ${ }^{4}$ St Michael's Hospital, Toronto, Canada; ${ }^{5}$ Children's Hospital Fudan, Shanghai, China;

${ }^{6}$ Public Health Agency of Canada, Ottawa, Canada

\subsection{6/archdischild-2018-rcpch.482}

Background and objectives Universal hypoglycaemia monitoring of newborns is not recommended. We wished to determine the incidence, presentation and case characteristics of hypoglycaemic newborns that were not being monitored.

Methods Through the Canadian Paediatric Surveillance Program we conducted a national study of severe hypoglycaemia in apparently low-risk full-term newborns. Inclusion criteria were: an otherwise healthy infant less than 96 hours old; gestational age 37-42 weeks; birth weight 2500-3999 grams; whole blood or serum glucose less than $2.0 \mathrm{mmol} / \mathrm{L}$ and IV dextrose used to treat the hypoglycaemia. Data were managed and analysed using IBM SPSS Statistics for Windows, Version 24.0 (Armonk, NY: IBM Corp.).

Results From April 2014 to March 2016, 177 cases were reported. There were 5 duplicates, 33 cases did not meet inclusion criteria and 46 questionnaires were not returned, leaving 93 confirmed cases. The estimated incidence was 1 in 8378 births. All cases were singletons, 56\% were first-borns and $65 \%$ were male. An $8 \%$ rate of First Nations cases was 3 -fold the population rate. Maternal hypertension was present in 23\%, 4-fold the overall pregnancy rate. Maternal obesity was double the overall pregnancy rate at 23\%. Concerning signs or feeding issues were present at diagnosis in $98 \%$. Median time to diagnosis was 4.1 hours. Mean blood glucose was $1.4 \pm 0.5$ hours (SD). Seventy eight percent had at least one of 4 potential perinatal stress indicators (emergency Caesarean Section, meconium at delivery, requiring resuscitation or cord artery $\mathrm{pH}<7.10)$. Those cases were more likely to be diagnosed before 6 hours $(p=0.03)$. Twenty five percent (23 cases) were small for gestational age (SGA) with birth weight $<10$ th centile, of which 5 had seizures and 5 had hyperinsulinism. Presentation with major clinical signs (seizure, apnoea or cyanosis) occurred in 20\%. Neurodevelopmental concern was present in $20 \%$ of all cases. Amongst 13 cases which had brain MRI, 11 were abnormal.

Conclusion While acknowledging the study's limitations, the impact of First Nations origin, maternal obesity, maternal hypertension and perinatal stress indicators warrant further study and possible incorporation into glucose monitoring guidelines. The data further supports adoption of norm-based standards to identify and monitor all SGA infants.

Funding Supported by grants from Queen's University Faculty of Health Sciences and the Public Health Agency of Canada.

\section{TEN YEARS OF ALCOHOL INTOXICATIONS IN ADOLESCENTS AND TREATMENT IN PAEDIATRIC DEPARTMENTS IN DUTCH HOSPITALS}

${ }^{1} \mathrm{~N}$ Van Der Lely, ${ }^{2} \mathrm{JJV}$ Hoof, ${ }^{1} \mathrm{I}$ Wolberink, ${ }^{1} \mathrm{~K}$ Nienhuis. ${ }^{1}$ Reinier de Graaf Gasthuis, Delft, The Netherlands; ${ }^{2}$ Behavioral Sciences Faculty, University of Twente, Enschede, The Netherlands
Aim Alcohol intoxication in children and adolescents is a severe health concern in current paediatrics. In this longitudinal study we monitored intake and treatment of 5893 adolescents in Departments of Paediatrics in Dutch hospitals over the years 2007 to 2016 .

Methods from 2007 till 2016 we collected data on all adolescents (inclusion criteria: aged younger than 18 and with a positive blood alcohol concentration (BAC), treated by a paediatrician in all Dutch hospitals. Within the Dutch Paediatric Surveillance System (NSCK), all paediatricians report adolescents and fill in a questionnaire, making use of a patient interview.

Results in total 5893 adolescents were treated, mainly $(4,678$; $88 \%)$ related to severe alcohol intoxication; mean age was 15.4 years, and 52\% were boys. BAC level increased during this period (1.82 in 2007 and 2.01 in 2016), and reduced consciousness lasted from 2.24 hours in 2007 till 3.12 hours in 2016). 11.4\% Of the adolescents with alcohol intoxication had simultaneous drug usage. The attitude of the parents changed during the years: in 2011 (first year of registration) $68 \%$ of the parents gave permission to their child to drink alcohol, in 2016 this decreased to only $19 \%$.

Conclusions alcohol intoxication treatment remains an issue of importance. This dataset enables us to conduct longitudinal and interesting analyses on alcohol intoxication characteristics in youngsters, medical treatment, and events leading up to the intoxication.

\section{I16 NUTRITIONAL RICKETS PRESENTING TO SECONDARY CARE IN CHILDREN (<16 YEARS) - A UK SURVEILLANCE STUDY}

${ }^{1} \mathrm{P}$ Julies, ${ }^{2} \mathrm{RM}$ Lynn, ${ }^{2} \mathrm{~K}$ Pall, ${ }^{2} \mathrm{M}$ Leoni, ${ }^{3} \mathrm{~A}$ Calder, ${ }^{4} \mathrm{Z}$ Mughal, ${ }^{5} \mathrm{~N}$ Shaw, ${ }^{6} \mathrm{H}$ McDevitt, ${ }^{7} \mathrm{C}$ MacDonnell, ${ }^{8} \mathrm{M}$ Blair. ${ }^{1}$ Royal Free Hospital, London, UK; ${ }^{2}$ BPSU, London, UK; ${ }^{3}$ Great Ormond Street Hospital, London, UK; ${ }^{4}$ Manchester University Hospital, Manchester, UK; ${ }^{5}$ Birmingham Children's Hospital, Birmingham, UK; ${ }^{6}$ University of Glasgow, Glasgow, UK; ${ }^{7}$ National Children's Hospital, Tallaght, Republic of Ireland; ${ }^{8}$ Imperial College, London, UK

\subsection{6/archdischild-2018-rcpch.484}

Aims Rickets is a disease of growing children with serious short and long-term complications. Although the prevalence of rickets has been reported widely to be increasing the actual national incidence of nutritional rickets (NR) in the United Kingdom (UK) is unknown. This study aims to describe the incidence, presentation, and clinical management of children with NR in the UK and ROI.

Methods Data was collected prospectively monthly between March 2015-March 2017 from 3500 paediatricians using British Paediatric Surveillance Unit reporting methodology with the following definition (table 1):

Abstract I16 Table 1
Clinical rickets with any of the following:
- Leg deformity/Swollen wrists or knees or ribs AND 250 hour vitamin $D<25 \mathrm{nmol} / \mathrm{L}$ with
one or more abnormalities of serum calcium, alkaline phosphatase, phosphate,
parathyroid hormone
OR
Radiological rickets with:
- Widening, cupping, splaying of metaphysis (of any long bone) AND 250HVitamin
D<25 nmol/L

